\title{
Multi-level Strategy for Computer-Assisted Transbronchial Biopsy
}

\author{
Ivan Bricault ${ }^{1}$, Gilbert Ferretti ${ }^{2}$ and Philippe Cinquin ${ }^{1}$ \\ 1 TIMC-IMAG, Institut Albert Bonniot, 38706 La Tronche cedex, France \\ ${ }^{2}$ Department of Radiology, Hôpital Michallon, CHU Grenoble, France \\ E-mail : Ivan.Bricaultoimag.fr
}

\begin{abstract}
The Computer-Assisted Transbronchial Biopsy project involves the registration, without any external localization device, of a pre-operative 3D CT scan of the thoracic cavity (showing a tumor that requires a needle biopsy), and an intra-operative endoscopic $2 \mathrm{D}$ image sequence, in order to provide an assistance to a transbronchial puncture of the tumor. Because of the specific difficulties resulting from the processed data, original image processing methods were elaborated and a multi-level strategy is introduced. For each analysis level, the relevant information to process and the corresponding algorithms are defined. This multi-level strategy then achieves the best possible accuracy.

The results presented here demonstrate that it is possible to localize precisely the endoscopic camera within the CT data coordinate system. The computer can thus synthesize in near real-time the CT-derived virtual view that corresponds to the actual endoscopic real view.
\end{abstract}

\section{Introduction}

For diagnosing and staging lung cancers in patients, obtaining histological samples is a fundamental step. But many mediastinal tumor formations are invisible during bronchoscopy and not accessible to a transthoracic biopsy. Then a transbronchial needle biopsy is a minimally invasive alternative to surgery, and thus a very valuable procedure [1].

Nevertheless, the transbronchial biopsy procedure involves a "blind" puncture without a direct visual control on the lesion. So, when no specific assistance is provided, it can explain that despite of its potential benefits the transbronchial biopsy remains underutilized [2].

A computer assistance can promote the transbronchial biopsy. The computer can reconstruct 3-D endoluminal views from CT data (Virtual Bronchoscopy). When preparing a transbronchial biopsy, virtual views can help a pre-operative planning of an optimal needle trajectory [3].

But virtual bronchoscopy provides only a pre-operative assistance. The aim of Computer-Assisted Transbronchial Biopsy is to provide an intra-operative assistance : during the endoscopic procedure, the operator wants to see in real-time the exact virtual view (computed from CT data) that corresponds to the current endoscopic real view, i.e. the virtual view must follow the movements of the fiberoptic camera inside bronchial tree. Thus, the operator benefits from an Augmented Reality technique : extraluminal elements detected on CT are augmenting the endoscopic perception (Figure 1). One can then verify that the needle reaches the tumor, without hurting any critical tissues. 


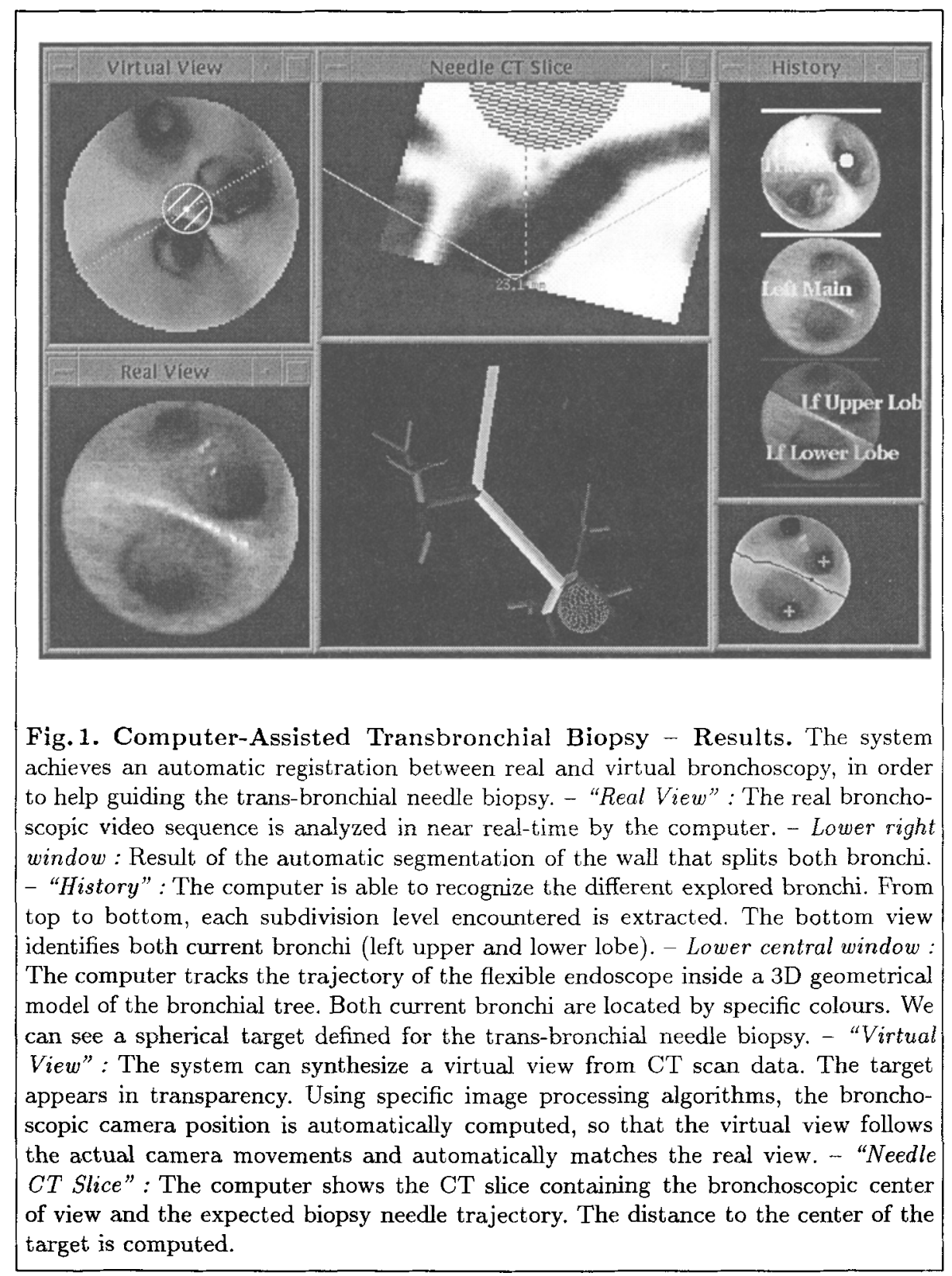

\section{A multi-level strategy}

Our approach is based on algorithms able to localize in real-time the fiberoptic camera position inside the CT data coordinate system, using only image processing, and without any external localization device. Because this localization task is difficult, we have developed a specific multi-level strategy. Four levels of image analysis are required in order to achieve a complete localization of the endoscope. 
The level-1 analysis follows the endoscope movements inside the tracheobronchial tree hierarchy. The aim is to anatomically identify the various explored bronchial subdivisions, in order to help the operator navigate and find a correct path to the biopsy site.

Then, when the operator is in the neighborhood of the lesion, an accurate localization of the endoscope is achieved in levels $2,3,4$ in order to guide the biopsy in a safe and accurate manner :

- The level-2 analysis gives an approximative endoscopic localization, producing a virtual view roughly similar to the real view.

- The level-3 analysis generates a precise endoscopic localization, but is restricted to 4 degrees-of-freedom.

- Finally, the level-4 analysis computes an endoscopic localization with 6 degrees-of-freedom ( 3 translations and 3 rotations entirely define the endoscopic camera position in the CT coordinate system).

\subsection{Level-1 : Bronchi anatomical identification}

We have achieved the bronchial subdivision image segmentation with mathematical morphology operators, involving dynamics and watershed line computations (see [4] for details).

A 3D geometrical model of the tracheo-bronchial tree allows us to predict the various subdivision relative orientations. During a real endoscopic exploration, it is then possible to identify each bronchus thanks to a comparison between segmented (endoscopic) and expected (CT) subdivision orientations. We need to use only one generic 3D bronchial model for every patients : indeed, our method is robust enough to tolerate the small inter-individual anatomical variations.

Using a digital alpha workstation and $100 \times 100$ endoscopic images, the computer can process 2 to 10 images per second. The computer is able to follow an endoscopic exploration up to the fourth level of subdivision.

\subsection{Level-2 : Approximative localization}

We consider here that the direction of the endoscopic axis of view remains parallel to the bronchial axis. Level-2 registration uses the alignment between CT and endoscopic inter-bronchial walls (see [4] for details). It is a direct localization method that involves no optimization process and that is very fast. The whole procedure, including endoscopic image segmentation and virtual image computation, allows a tracking of camera movements with 0.5 to 2 images per second.

The quality of the real/virtual registration depends on the fitting between CT-defined and segmented inter-bronchial walls, and depends on the parallelism of CT-defined bronchial axis and actual endoscopic axis of view. As we will describe it in the following, when these conditions are not fulfilled, then level-3 and level-4 are useful to reestablish the matching quality.

\subsection{Level-3 : four degrees-of-freedom precise localization}

We use here a new 2D/2D "daemon-based" [5] registration algorithm, presented in [6]. We still suppose, here, that the endoscope axis remains aligned with the bronchial axis (4 degrees of freedom). 
We try to find the virtual view that best matches an endoscopic real view. An initial virtual view is computed from level-2 algorithm. From the translation, rotation and zoom parameters given by real/virtual "daemon-based" registration, we can adjust the estimated endoscope position and then create a new virtual view. Iterating this process can finally give a virtual view very similar to real view : it means that the endoscope is correctly located [6]. We usually achieve the level-3 localization with 2 or 3 iterations only and no more than a few seconds.

\subsection{Level-4 : six degrees-of-freedom localization}

As level-3 registration involves an intermediate $2 \mathrm{D} / 2 \mathrm{D}$ registration step, the camera localization is restricted to four degrees of freedom. In this section, we present a precise six degrees-of-freedom localization that involves a $3 \mathrm{D}$ reconstruction of bronchial surface, thanks to a model-based shape-from-shading algorithm.

Motivations Level-3 algorithm needs to be very tolerant against pixel intensity variations. Indeed, when we try to register virtual and real views, we want to consider only the structural information in the images (i.e. the global shape of bronchial subdivisions). Thus our daemon-based algorithm, involving only unitary vectors in the direction of decreasing grey values [6] is very robust regarding lightening conditions : as a characteristic of our multi-level strategy, for a particular analysis level, we select all the relevant information but only the relevant information.

Nevertheless, pixel intensity values are important, because they can reveal a difference between real and virtual optical axis directions : when optical axis direction changes, some parts of the image become darker while others become brighter. As we will demonstrate it in the following, thanks to a "model-based shape-from-shading", we will be able to process these grey value informations, but only because level-3 gives us the necessary pre-registered initial conditions.

In the case of bronchoscopic images, the shape-from-shading problem is very difficult, because we have to tackle major topological ambiguities :

- only a small part of bronchial surface appears in one bronchoscopic image.

The information found in the image is not sufficient to solve the topological ambiguities (in particular concerning the image edges, which represent in reality the major part of the image).

- in a bronchial subdivision image, the only visible singular point is a saddle point, so it can not be used for propagation [7]. We should use a propagation from the extremity of both bronchi, but these regions are not correctly visualized (occultation, lightening attenuation).

Furthermore, we have to consider a point light source at finite distance, and a perspective projection. Only few algorithms studied such image construction conditions [8] (but they do not have to tackle topological ambiguities).

Finally, the difficulties encountered for bronchial image 3D reconstruction are numerous and can not be solved by usual shape-from-shading algorithms developed so far. 
(a)

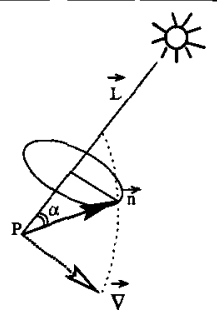

(b)

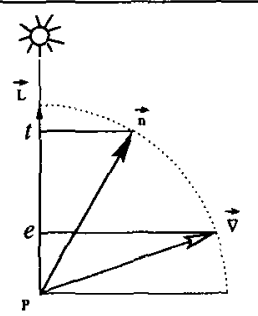

Fig. 2. Using model-based constraints for shape-from-shading. (a) The bronchial surface normal $\mathbf{n}$, corresponding to an endoscopic pixel with intensity equal to $t=\cos (\alpha)$, has an undefined position on the cone forming an angle $\alpha$ with the lightening direction $\mathbf{L}$. Knowing the $\mathrm{CT}$ surface normal $\nabla$, we can choose $\mathbf{n}$ as the vector on the cone that is the closest to $\nabla$. (b) $\mathbf{n}$ can then be computed from $t, \nabla$, and $e$ which is the pixel intensity in the CT virtual image (see text).

Model-based shape-from-shading Our solution comes from the development of a new shape-from-shading algorithm : we use the $3 \mathrm{D}$ data about the bronchial surface (coordinates, distance to camera, normal vectors) that are given by the pre-registered CT model resulting from level-3. Our algorithm is based on the 3 following points.

(1) CT-derived 3D data allows us to correct lightening attenuation effects. During the synthesis of virtual images, the lightening attenuation is computed from the distance of the bronchial point to light source. Considering any pixel in a real bronchial image, an approximation of this distance to light source is given by the pre-registered 3D CT model. Then we can use the lightening attenuation formula, in order to correct lightening artifacts before processing the image.

(2) CT data allows us to compute real bronchi normals. Let us consider a pixel in a bronchial subdivision real image. Let $t$ be the pixel intensity (from 0.0 to 1.0 ). If $\mathbf{n}$ is the unknown normal direction in the corresponding bronchial surface point, and $\mathbf{L}$ is the light ray direction, then $\mathbf{n}$ must be such that $t=\mathbf{n} \cdot \mathbf{L}$, but $\mathbf{n}$ remains undefined (Figure 2a).

Let $e$ be the same pixel intensity in the virtual image, previously registered (level-3) with the real image. We have $e=\boldsymbol{\nabla} \cdot \mathbf{L}$, where $\boldsymbol{\nabla}$ is the normal direction given by the 3D CT model in the corresponding bronchial surface point (Figure 2b).

Then we can decide to choose $\mathbf{n}$ as the vector, such that $\mathbf{n} \cdot \mathbf{L}=t$, which is the closest to $\boldsymbol{\nabla}$. So we can show easily that $\mathbf{n}$ is defined by

$$
\mathbf{n}=\left(t-e \sqrt{\frac{1-t^{2}}{1-e^{2}}}\right) \mathbf{L}+\sqrt{\frac{1-t^{2}}{1-e^{2}}} \boldsymbol{\nabla}
$$

(3) Eq.3 above defines the normal vectors for each pixel in bronchial image. Then the whole surface can be reconstructed, from the center of inter-bronchial wall (its coordinates are given by $3 \mathrm{D} \mathrm{CT}$ model), propagating surface variations given by normal vectors and using a $3 \mathrm{D}$ grid model of the reconstructed surface ( $3 \mathrm{D}$ because of the specificities of the perspective projection).

Level-4 CT/bronchoscopy registration Up to level-3, the optical axis was previously maintained fixed when estimating the camera position. We now want 


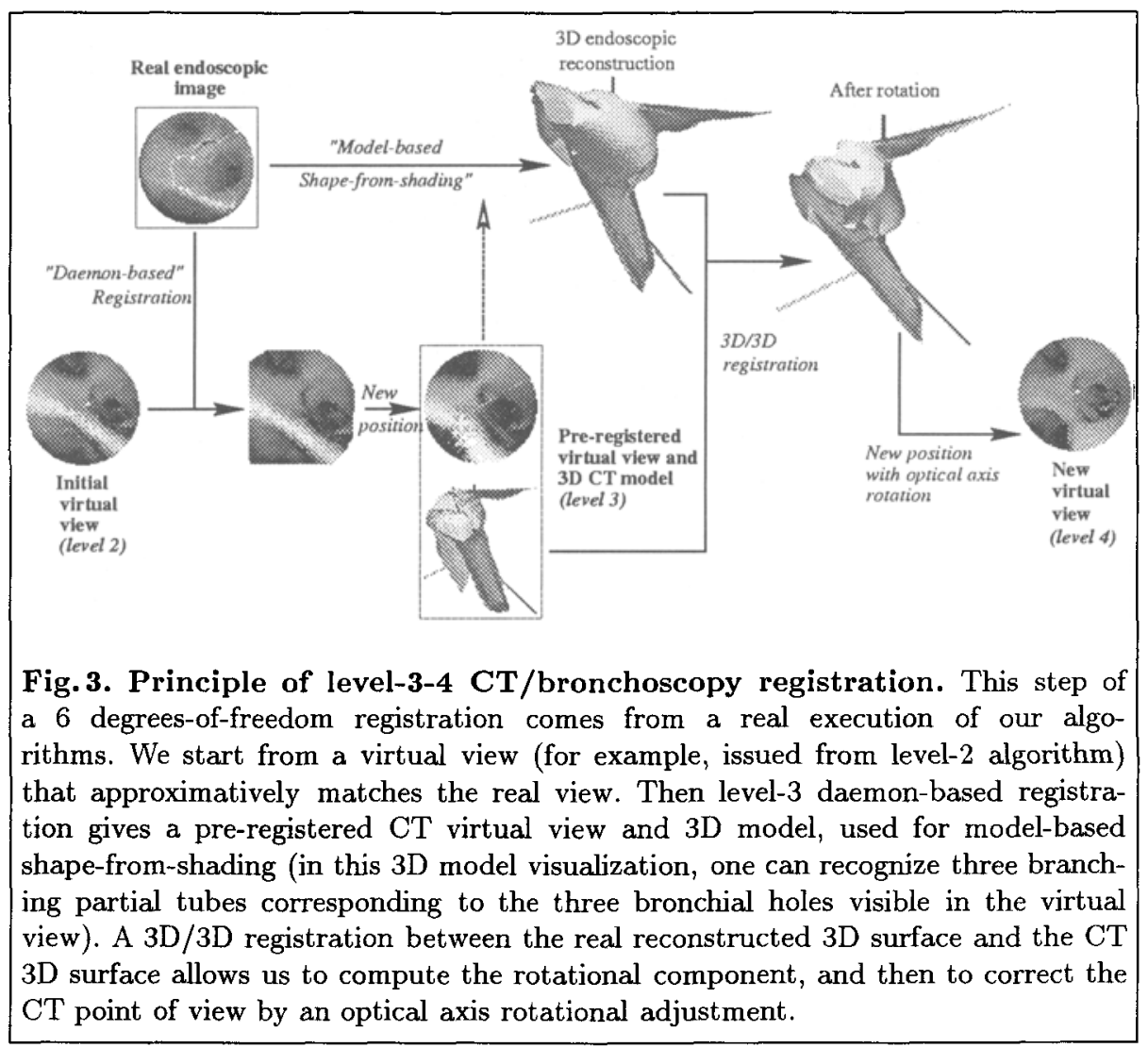

to adjust an incorrect optical axis direction estimation, i.e. we want to achieve a full 6 degree-of-freedom $\mathrm{CT} /$ bronchoscopy registration.

We use here the 3D bronchial surface resulting from our model-based shapefrom-shading algorithm. This endoscopic 3D surface can be compared to CT model 3D surface. A least-squares fitting of these two 3D point sets is achieved; for that purpose, $3 \mathrm{D}$ points from each set corresponding to the same light ray are matched. The rotation between CT model surface and endoscopic reconstructed surface, given by this $3 \mathrm{D} / 3 \mathrm{D}$ registration step, is then applied to optical axis direction (Figure 3). As a result, the difference between virtual and real optical axis directions decreases.

Such a registration process can be iterated : the level-2 initial virtual view is replaced by the new corrected virtual view, and level-3 and level-4 processings are iterated.

Results Our model-based shape-from-shading method is able to take into account lightening and perspective artifacts, and to solve topological ambiguities, thanks to CT data.

Iterating the Figure 3 localization process, in general only twice, we obtained on real endoscopic images very good localization results, with a computation time being no more than a few seconds. Tests with virtual images show the good results obtained concerning the registration accuracy (Figure 4). We observe 


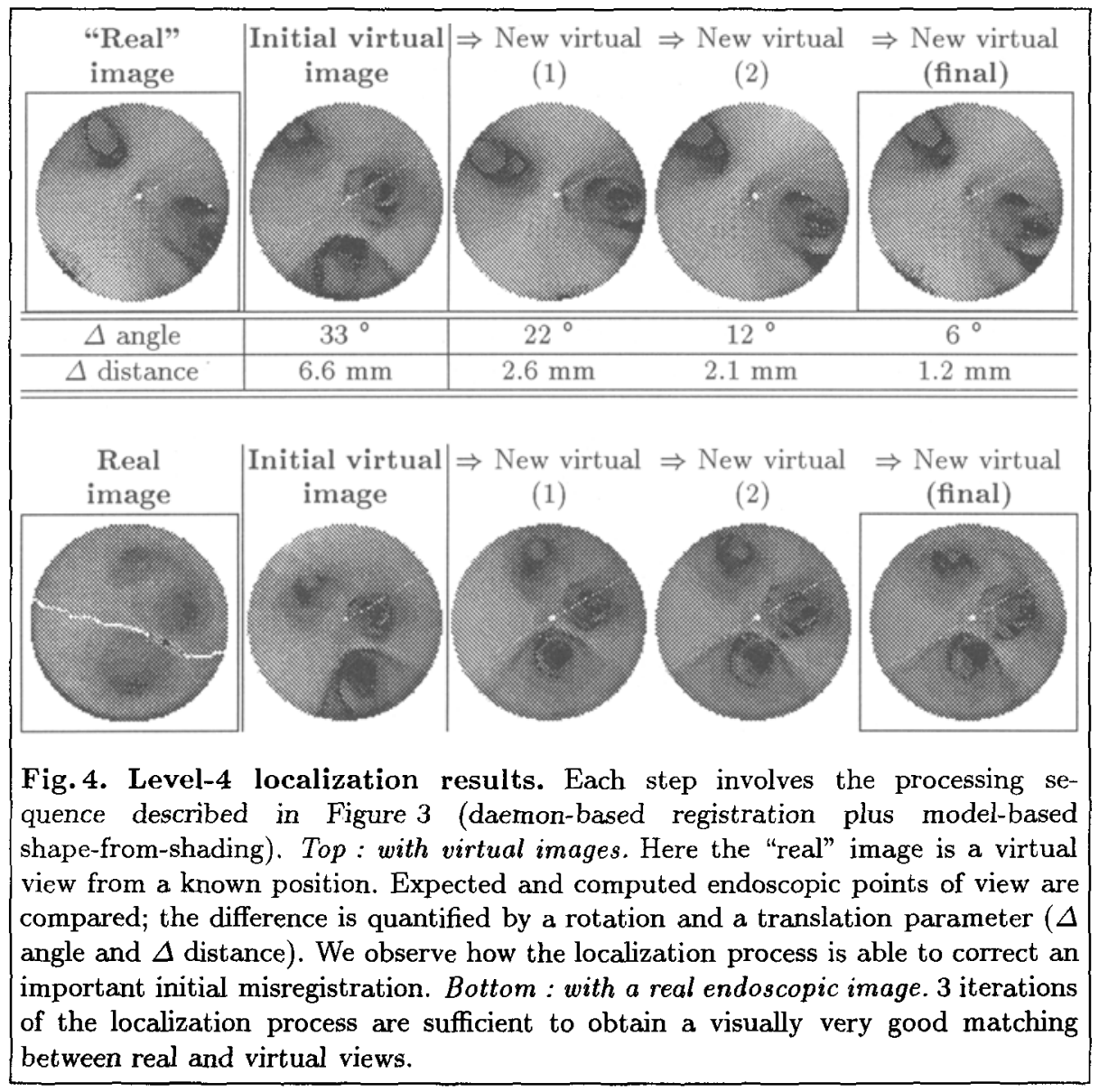

that the achieved precision increases during level-4 process. The demonstrated final precision is sufficient for clinical applications, considering that the tumor diameter is generally greater than 10 millimeters.

A failure can occur if the reference real image or a generated virtual image happen to be not informative enough (for example if the endoscope is too close to the bronchial wall, or if bronchial edges are too partially visualized). Nevertheless, in case of failure, our multi-level strategy leaves the possibility to restrict the analysis to level-2. Furthermore, this level-2 localization can benefit from adjusted parameters (optical axis direction, CT inter-bronchial wall geometry) inherited from a level-3 and level-4 analysis on a previous image with better quality.

\section{Conclusion}

Computer-assisted transbronchial biopsy is a challenging medical and scientific issue. As far as we know, our prototype system constitutes the first proposed solution : indeed, bronchoscopic data can be hardly processed by most usual computer vision algorithms, because of many specific difficulties. 
We divided this complex problem into four levels, with an increasing analysis accuracy, and we selected for each level the relevant information and methods. So the success of our approach is based on the introduction of this multi-level modelbased image analysis strategy, together with various innovative algorithms.

With our computer-assisted transbronchial biopsy system, execution speeds are below 5 seconds for level 1 to 4 complete $100 \times 100$ image processing. Accuracy, measured by using virtual instead of real images, is about 1 to 2 millimeters and 5 degrees. This is compatible with medical applications, considering tumoral formations typically greater than 10 millimeters. Tests on real endoscopic data show that the real/virtual registration result is good and can be very informative for the transbronchial biopsy assistance.

Finally, let us note that the current state of this work uses endoscopic video sequences, stored on the computer, then a posteriori processed. Nevertheless, the algorithms execution speed demonstrates the real-time capability of our system. This is confirmed by preliminary real-time tests using a tracheobronchial anatomical specimen. In the near future, we will proceed into further experiments in clinical conditions, and we will develop dedicated protocols in order to fully evaluate our system efficiency and reliability.

Acknowledgments : This work was supported in part by the French "Ligue Nationale contre le Cancer", Comité de l'Isère.

\section{References}

1. J. F. Turner and Ko-Pen Wang, "Staging of mediastinal involvement in lung cancer by bronchoscopic needle aspiration," Journal of Bronchology, vol. 3, pp. 74-76, 1996.

2. Edward F. Haponik and Deborah Shure, "Underutilization of transbronchial needle aspiration," Chest, vol. 112, no. 1, july 1997 .

3. H.P. McAdams, P.C. Goodman, and P. Kussin, "Virtual bronchoscopy for directing transbronchial needle aspiration of malignant hilar and mediastinal lymph nodes," in Radiological Society of North America, Chicago, 1996.

4. Ivan Bricault, Gilbert Ferretti, and Philippe Cinquin, "Computer-Assisted Bronchoscopy : Aims and Research Perspectives," Computer Aided Surgery (formerly : Journal of Image Guided Surgery), vol. 1, no. 4, pp. 217-225, 1995, [Also in MRCAS'95 proceedings].

5. Jean-Philippe Thirion, "Fast intensity based non rigid matching," in Medical Robotics and Computer assisted Surgery, Baltimore, november 1995, pp. 47-54.

6. Ivan Bricault, "A Fast Morphology-Based Registration - Application to ComputerAssisted Bronchoscopy," in CVRMed-MRCAS, J. Troccaz, E. Grimson, and R. Mösges, Eds. march 1997, Lecture Notes in Computer Science, Springer-Verlag.

7. Ron Kimmel and Alfred M. Bruckstein, "Global Shape from Shading," Computer Vision and Image Understanding, vol. 62, no. 3, pp. 360-369, november 1995.

8. Takayuki Okatani and Koichiro Deguchi, "Shape Reconstruction from an Endoscope Image by Shape from Shading Technique for a Point Light Source at the Projection Center," Computer Vision and Image Understanding, vol. 66, no. 2, pp. 119-131, may 1997 . 